\title{
Quantum Deformations and Superintegrable Motions on Spaces with Variable Curvature
}

\author{
Orlando RAGNISCO ${ }^{\dagger}$, Ángel BALLESTEROS ${ }^{\ddagger}$, Francisco J. HERRANZ ${ }^{\ddagger}$ and Fabio MUSSO ${ }^{\dagger}$ \\ † Dipartimento di Fisica, Università di Roma Tre and Instituto Nazionale di Fisica Nucleare \\ sezione di Roma Tre, Via Vasca Navale 84, I-00146 Roma, Italy \\ E-mail: ragnisco@fis.uniroma3.it,musso@fis.uniroma3.it \\ ¥Departamento de Física, Universidad de Burgos, E-09001 Burgos, Spain \\ E-mail: angelb@ubu.es, fjherranz@ubu.es
}

Received November 12, 2006, in final form January 22, 2007; Published online February 14, 2007

Original article is available at http://www.emis.de/journals/SIGMA/2007/026/

\begin{abstract}
An infinite family of quasi-maximally superintegrable Hamiltonians with a common set of $(2 N-3)$ integrals of the motion is introduced. The integrability properties of all these Hamiltonians are shown to be a consequence of a hidden non-standard quantum $s l(2, \mathbb{R})$ Poisson coalgebra symmetry. As a concrete application, one of this Hamiltonians is shown to generate the geodesic motion on certain manifolds with a non-constant curvature that turns out to be a function of the deformation parameter $z$. Moreover, another Hamiltonian in this family is shown to generate geodesic motions on Riemannian and relativistic spaces all of whose sectional curvatures are constant and equal to the deformation parameter $z$. This approach can be generalized to arbitrary dimension by making use of coalgebra symmetry.
\end{abstract}

Key words: integrable systems; quantum groups; curvature; contraction; harmonic oscillator; Kepler-Coulomb; hyperbolic; de Sitter

2000 Mathematics Subject Classification: 37J35; 17B37

\section{Introduction}

The set of known maximally superintegrable systems on the $N$-dimensional (ND) Euclidean space is very limited: it comprises the isotropic harmonic oscillator with $N$ centrifugal terms (the so-called Smorodinsky-Winternitz (SW) system [1, 2]), the Kepler-Coulomb (KC) problem with $(N-1)$ centrifugal barriers [3] (and some symmetry-breaking generalizations of it [4]), the Calogero-Moser-Sutherland model [5, 6, 7, 8] and some systems with isochronous potentials [9]. Both the SW and the KC systems have integrals quadratic in the momenta, and also both of them have been generalized to spaces with non-zero constant curvature (see $[10,11,12,13$, $14,15,16,17,18,19,20])$. In order to complete this brief ND summary, Benenti systems on constant curvature spaces have also to be considered [21], as well as a maximally superintegrable deformation of the SW system that was introduced in [22] by making use of quantum algebras.

More recently, the study of $2 \mathrm{D}$ and $3 \mathrm{D}$ superintegrable systems on spaces with variable curvature has been addressed [23, 24, 25, 26, 27, 28, 29]. The aim of this paper is to give a general setting, based on quantum deformations, for the explicit construction of certain classes of superintegrable systems on $N D$ spaces with variable curvature.

In order to fix language conventions, we recall that an ND completely integrable Hamiltonian $H^{(N)}$ is called maximally superintegrable (MS) if there exists a set of $(2 N-2)$ globally

${ }^{\star}$ This paper is a contribution to the Proceedings of the O'Raifeartaigh Symposium on Non-Perturbative and Symmetry Methods in Field Theory (June 22-24, 2006, Budapest, Hungary). The full collection is available at http://www.emis.de/journals/SIGMA/LOR2006.html 
defined functionally independent constants of the motion that Poisson-commute with $H^{(N)}$. Among them, at least two different subsets of $(N-1)$ constants in involution can be found. In the same way, a system will be called quasi-maximally superintegrable (QMS) if there are $(2 N-3)$ integrals with the abovementioned properties. All MS systems are QMS ones, and the latter have only one less integral than the maximum possible number of functionally independent ones.

In this paper we present the construction of QMS systems on variable curvature spaces which is just the quantum algebra generalization of a recent approach to ND QMS systems on constant curvature spaces that include the SW and $\mathrm{KC}$ as particular cases [30]. Some of these variable curvature systems in 2D and 3D have been already studied (see [31,32, 33]), and we present here the most significant elements for their $N D$ generalizations. We will show that this scheme is quite efficient in order to get explicitly a large family of QMS systems. Among them, some specific choices for the Hamiltonian can lead to a MS system, for which only the remaining integral has to be explicitly found.

In the the next Section we will briefly summarize the $N D$ constant curvature construction given in [30], that makes use of an $s l(2, \mathbb{R})$ Poisson coalgebra symmetry. The generic variable curvature approach will be obtained in Section 3 through a non-standard quantum deformation of an $\operatorname{sl}(2, \mathbb{R})$ Poisson coalgebra. Some explicit $2 \mathrm{D}$ and $3 \mathrm{D}$ spaces defined through free motion Hamiltonians will be given in Section 4, and the ND generalization of them will be sketched in Section 5. Section 6 is devoted to the introduction of some potentials that generalize the KC and SW ones. A final Section including some comments and open questions closes the paper.

\section{QMS Hamiltonians with $s l(2, \mathbb{R})$ coalgebra symmetry}

Let us briefly recall the main result of [30] that provides an infinite family of QMS Hamiltonians. We stress that, although some of these Hamiltonians can be interpreted as motions on spaces with constant curvature, this approach to QMS systems is quite general, and also nonnatural Hamiltonian systems (for instance, those describing static electromagnetic fields) can be obtained.

Theorem 1 ([30]). Let $\{\mathbf{q}, \mathbf{p}\}=\left\{\left(q_{1}, \ldots, q_{N}\right),\left(p_{1}, \ldots, p_{N}\right)\right\}$ be $N$ pairs of canonical variables. The ND Hamiltonian

$$
H^{(N)}=\mathcal{H}\left(\mathbf{q}^{2}, \tilde{\mathbf{p}}^{2}, \mathbf{q} \cdot \mathbf{p}\right),
$$

with $\mathcal{H}$ any smooth function and

$$
\mathbf{q}^{2}=\sum_{i=1}^{N} q_{i}^{2}, \quad \tilde{\mathbf{p}}^{2}=\sum_{i=1}^{N}\left(p_{i}^{2}+\frac{b_{i}}{q_{i}^{2}}\right) \equiv \mathbf{p}^{2}+\sum_{i=1}^{N} \frac{b_{i}}{q_{i}^{2}}, \quad \mathbf{q} \cdot \mathbf{p}=\sum_{i=1}^{N} q_{i} p_{i},
$$

where $b_{i}$ are arbitrary real parameters, is $Q M S$. The $(2 N-3)$ functionally independent and "universal" integrals of motion are explicitly given by

$$
\begin{aligned}
& C^{(m)}=\sum_{1 \leq i<j}^{m}\left\{\left(q_{i} p_{j}-q_{j} p_{i}\right)^{2}+\left(b_{i} \frac{q_{j}^{2}}{q_{i}^{2}}+b_{j} \frac{q_{i}^{2}}{q_{j}^{2}}\right)\right\}+\sum_{i=1}^{m} b_{i}, \\
& C_{(m)}=\sum_{N-m+1 \leq i<j}^{N}\left\{\left(q_{i} p_{j}-q_{j} p_{i}\right)^{2}+\left(b_{i} \frac{q_{j}^{2}}{q_{i}^{2}}+b_{j} \frac{q_{i}^{2}}{q_{j}^{2}}\right)\right\}+\sum_{i=N-m+1}^{N} b_{i},
\end{aligned}
$$

where $m=2, \ldots, N$ and $C^{(N)}=C_{(N)}$. Moreover, the sets of $N$ functions $\left\{H^{(N)}, C^{(m)}\right\}$ and $\left\{H^{(N)}, C_{(m)}\right\}(m=2, \ldots, N)$ are in involution. 
The proof of this general result is based on the observation that, for any choice of the function $\mathcal{H}$, the Hamiltonian $H^{(N)}$ has an $s l(2, \mathbb{R})$ Poisson coalgebra symmetry [34] generated by the following Lie-Poisson brackets and comultiplication map:

$$
\begin{aligned}
& \left\{J_{3}, J_{+}\right\}=2 J_{+}, \quad\left\{J_{3}, J_{-}\right\}=-2 J_{-}, \quad\left\{J_{-}, J_{+}\right\}=4 J_{3}, \\
& \Delta\left(J_{l}\right)=J_{l} \otimes 1+1 \otimes J_{l}, \quad l=+,-, 3 .
\end{aligned}
$$

The Casimir function for $\operatorname{sl}(2, \mathbb{R})$ reads

$$
\mathcal{C}=J_{-} J_{+}-J_{3}^{2} \text {. }
$$

In fact, the coalgebra approach [34] provides an $N$-particle symplectic realization of $\operatorname{sl}(2, \mathbb{R})$ through the $N$-sites coproduct of $(2.4)$ living on $\operatorname{sl}(2, \mathbb{R}) \otimes \ldots N) \otimes \operatorname{sl}(2, \mathbb{R})[22]$ :

$$
J_{-}=\sum_{i=1}^{N} q_{i}^{2} \equiv \mathbf{q}^{2}, \quad J_{+}=\sum_{i=1}^{N}\left(p_{i}^{2}+\frac{b_{i}}{q_{i}^{2}}\right) \equiv \mathbf{p}^{2}+\sum_{i=1}^{N} \frac{b_{i}}{q_{i}^{2}}, \quad J_{3}=\sum_{i=1}^{N} q_{i} p_{i} \equiv \mathbf{q} \cdot \mathbf{p},
$$

where $b_{i}$ are $N$ arbitrary real parameters. This means that the $N$-particle generators (2.6) fulfil the commutation rules (2.3) with respect to the canonical Poisson bracket. As a consequence of the coalgebra approach, these generators Poisson commute with the $(2 N-3)$ functions $(2.2)$ given by the sets $C^{(m)}$ and $C_{(m)}$, which are obtained, in this order, from the "left" and "right" $m$-th coproducts of the Casimir (2.5) with $m=2,3, \ldots, N$ (see [35] for details). Therefore, any arbitrary function $\mathcal{H}$ defined on the $N$-particle symplectic realization of $s l(2, \mathbb{R})(2.6)$ is of the form (2.1), that is,

$$
H^{(N)}=\mathcal{H}\left(J_{-}, J_{+}, J_{3}\right)=\mathcal{H}\left(\mathbf{q}^{2}, \mathbf{p}^{2}+\sum_{i=1}^{N} \frac{b_{i}}{q_{i}^{2}}, \mathbf{q} \cdot \mathbf{p}\right),
$$

and defines a QMS Hamiltonian system that Poisson-commutes with all the "universal integrals" $C^{(m)}$ and $C_{(m)}$.

Notice that for arbitrary $N$ there is a single constant of the motion left to assure maximal superintegrability. In this respect, we stress that some specific choices of $\mathcal{H}$ comprise maximally superintegrable systems as well, but the remaining integral does not come from the coalgebra symmetry and has to be deduced by making use of alternative procedures.

Let us now give some explicit examples of this construction.

\subsection{Free motion on Riemannian spaces of constant curvature}

It is immediate to realize that the kinetic energy $\mathcal{T}$ of a particle on the ND Euclidean space $\mathbf{E}^{N}$ directly arises through the generator $J_{+}$in the symplectic realization (2.6) with all $b_{i}=0$ :

$$
\mathcal{H}=\mathcal{T}=\frac{1}{2} J_{+}=\frac{1}{2} \mathbf{p}^{2} .
$$

Now the interesting point is that the kinetic energy on ND Riemannian spaces with constant curvature $\kappa$ can be expressed in Hamiltonian form as a function of the ND symplectic realization of the $\operatorname{sl}(2, \mathbb{R})$ generators $(2.6)$. In fact, this can be done in two different ways [30]:

$$
\begin{aligned}
& \mathcal{H}^{\mathrm{P}}=\mathcal{T}^{\mathrm{P}}=\frac{1}{2}\left(1+\kappa J_{-}\right)^{2} J_{+}=\frac{1}{2}\left(1+\kappa \mathbf{q}^{2}\right)^{2} \mathbf{p}^{2}, \\
& \mathcal{H}^{\mathrm{B}}=\mathcal{T}^{\mathrm{B}}=\frac{1}{2}\left(1+\kappa J_{-}\right)\left(J_{+}+\kappa J_{3}^{2}\right)=\frac{1}{2}\left(1+\kappa \mathbf{q}^{2}\right)\left(\mathbf{p}^{2}+\kappa(\mathbf{q} \cdot \mathbf{p})^{2}\right) .
\end{aligned}
$$

The function $\mathcal{H}^{\mathrm{P}}$ is just the kinetic energy for a free particle on the spherical $\mathbf{S}^{N}(\kappa>0)$ and hyperbolic $\mathbf{H}^{N}(\kappa<0)$ spaces when this is expressed in terms of Poincaré coordinates $\mathbf{q}$ and canonical momenta $\mathbf{p}$ (coming from a stereographic projection in $\mathbb{R}^{N+1}$ ); on the other hand $\mathcal{H}^{\mathrm{B}}$ corresponds to Beltrami coordinates and momenta (central projection). By construction, both Hamiltonians are QMS ones since they Poisson-commute with the integrals (2.2). 


\subsection{Superintegrable potentials on Riemannian spaces of constant curvature}

QMS potentials $\mathcal{V}$ on constant curvature spaces can now be constructed by adding some suitable functions depending on $J_{-}$to $(2.7)$ and by considering arbitrary centrifugal terms that come from symplectic realizations of the $J_{+}$generator with generic $b_{i}$ 's:

$$
\mathcal{H}=\mathcal{T}\left(J_{+}, J_{-}, J_{3}\right)+\mathcal{V}\left(J_{-}\right) .
$$

The Hamiltonians that we will obtain in this way are the curved counterpart of the Euclidean systems, and through different values of the curvature $\kappa$ we will simultaneously cover the cases $\mathbf{S}^{N}$ $(\kappa>0), \mathbf{H}^{N}(\kappa<0)$, and $\mathbf{E}^{N}(\kappa=0)$.

In order to motivate the choice of the potential functions $\mathcal{V}\left(J_{-}\right)$, it is important to recall that in the constant curvature analogues of the oscillator and $\mathrm{KC}$ problems the Euclidean radial distance $r$ is just replaced by the function $\frac{1}{\sqrt{\kappa}} \tan (\sqrt{\kappa} r)$ (see [30] for the expression of this quantity in terms of Poincaré and Beltrami coordinates). Also, for the sake of simplicity, the centrifugal terms coming from the symplectic realization with arbitrary $b_{i}$ will be expressed in ambient coordinates $x_{i}[30]$ :

$$
\text { Poincaré: } \quad x_{i}=\frac{2 q_{i}}{1+\kappa \mathbf{q}^{2}} ; \quad \text { Beltrami: } \quad x_{i}=\frac{q_{i}}{\sqrt{1+\kappa \mathbf{q}^{2}}} .
$$

Special choices for $\mathcal{V}\left(J_{-}\right)$lead to the following systems, that are always expressed in both Poincaré and Beltrami phase spaces:

- A curved Evans system. The constant curvature generalization of a 3D Euclidean system with radial symmetry [36] would be given by

$$
\begin{aligned}
& \mathcal{H}^{\mathrm{P}}=\mathcal{T}^{\mathrm{P}}+\mathcal{V}\left(\frac{4 J_{-}}{\left(1-\kappa J_{-}\right)^{2}}\right)=\frac{1}{2}\left(1+\kappa \mathbf{q}^{2}\right)^{2} \mathbf{p}^{2}+\mathcal{V}\left(\frac{4 \mathbf{q}^{2}}{\left(1-\kappa \mathbf{q}^{2}\right)^{2}}\right)+\sum_{i=1}^{N} \frac{2 b_{i}}{x_{i}^{2}}, \\
& \mathcal{H}^{\mathrm{B}}=\mathcal{T}^{\mathrm{B}}+\mathcal{V}\left(J_{-}\right)=\frac{1}{2}\left(1+\kappa \mathbf{q}^{2}\right)\left(\mathbf{p}^{2}+\kappa(\mathbf{q} \cdot \mathbf{p})^{2}\right)+\mathcal{V}\left(\mathbf{q}^{2}\right)+\sum_{i=1}^{N} \frac{b_{i}}{2 x_{i}^{2}},
\end{aligned}
$$

where $\mathcal{V}$ is an arbitrary smooth function that determines the central potential; the specific dependence on $J_{-}$of $\mathcal{V}$ corresponds to the square of the radial distance in each coordinate system.

- The curved Smorodinsky-Winternitz system [10, 11, 12, 13, 14, 15]. Such a system is just the Higgs oscillator $[16,17]$ with angular frequency $\omega$ (that arises as the argument of $\mathcal{V}$ in $(2.8)$ ) plus the corresponding centrifugal terms:

$$
\begin{aligned}
& \mathcal{H}^{\mathrm{P}}=\mathcal{T}^{\mathrm{P}}+\frac{4 \omega^{2} J_{-}}{\left(1-\kappa J_{-}\right)^{2}}=\frac{1}{2}\left(1+\kappa \mathbf{q}^{2}\right)^{2} \mathbf{p}^{2}+\frac{4 \omega^{2} \mathbf{q}^{2}}{\left(1-\kappa \mathbf{q}^{2}\right)^{2}}+\sum_{i=1}^{N} \frac{2 b_{i}}{x_{i}^{2}}, \\
& \mathcal{H}^{\mathrm{B}}=\mathcal{T}^{\mathrm{B}}+\omega^{2} J_{-}=\frac{1}{2}\left(1+\kappa \mathbf{q}^{2}\right)\left(\mathbf{p}^{2}+\kappa(\mathbf{q} \cdot \mathbf{p})^{2}\right)+\omega^{2} \mathbf{q}^{2}+\sum_{i=1}^{N} \frac{b_{i}}{2 x_{i}^{2}} .
\end{aligned}
$$

This is a MS Hamiltonian and the remaining constant of the motion can be chosen from any of the following $N$ functions:

$$
\begin{aligned}
& \mathcal{I}_{i}^{\mathrm{P}}=\left(p_{i}\left(1-\kappa \mathbf{q}^{2}\right)+2 \kappa(\mathbf{q} \cdot \mathbf{p}) q_{i}\right)^{2}+\frac{8 \omega^{2} q_{i}^{2}}{\left(1-\kappa \mathbf{q}^{2}\right)^{2}}+b_{i} \frac{\left(1-\kappa \mathbf{q}^{2}\right)^{2}}{q_{i}^{2}} \\
& \mathcal{I}_{i}^{\mathrm{B}}=\left(p_{i}+\kappa(\mathbf{q} \cdot \mathbf{p}) q_{i}\right)^{2}+2 \omega^{2} q_{i}^{2}+b_{i} / q_{i}^{2}, \quad i=1, \ldots, N
\end{aligned}
$$


- A curved generalized Kepler-Coulomb system [12, 13, 14, 18, 19, 20]. The curved KC potential with real constant $k$ together with $N$ centrifugal terms would be given by

$$
\begin{aligned}
& \mathcal{H}^{\mathrm{P}}=\mathcal{T}^{\mathrm{P}}-k\left(\frac{4 J_{-}}{\left(1-\kappa J_{-}\right)^{2}}\right)^{-1 / 2}=\frac{1}{2}\left(1+\kappa \mathbf{q}^{2}\right)^{2} \mathbf{p}^{2}-k \frac{\left(1-\kappa \mathbf{q}^{2}\right)}{2 \sqrt{\mathbf{q}^{2}}}+\sum_{i=1}^{N} \frac{2 b_{i}}{x_{i}^{2}}, \\
& \mathcal{H}^{\mathrm{B}}=\mathcal{T}^{\mathrm{B}}-k J_{-}^{-1 / 2}=\frac{1}{2}\left(1+\kappa \mathbf{q}^{2}\right)\left(\mathbf{p}^{2}+\kappa(\mathbf{q} \cdot \mathbf{p})^{2}\right)-\frac{k}{\sqrt{\mathbf{q}^{2}}}+\sum_{i=1}^{N} \frac{b_{i}}{2 x_{i}^{2}} .
\end{aligned}
$$

This is again a MS system provided that, at least, one $b_{i}=0$. In this case the remaining constant of the motion turns out to be

$$
\begin{aligned}
\mathcal{L}_{i}^{\mathrm{P}} & =\sum_{l=1}^{N}\left(p_{l}\left(1-\kappa \mathbf{q}^{2}\right)+2 \kappa(\mathbf{q} \cdot \mathbf{p}) q_{l}\right)\left(q_{l} p_{i}-q_{i} p_{l}\right)+\frac{k q_{i}}{2 \sqrt{\mathbf{q}^{2}}}-\sum_{l=1 ; l \neq i}^{N} b_{l} \frac{q_{i}\left(1-\kappa \mathbf{q}^{2}\right)}{q_{l}^{2}}, \\
\mathcal{L}_{i}^{\mathrm{B}} & =\sum_{l=1}^{N}\left(p_{l}+\kappa(\mathbf{q} \cdot \mathbf{p}) q_{l}\right)\left(q_{l} p_{i}-q_{i} p_{l}\right)+\frac{k q_{i}}{\sqrt{\mathbf{q}^{2}}}-\sum_{l=1 ; l \neq i}^{N} b_{l} \frac{q_{i}}{q_{l}^{2}} .
\end{aligned}
$$

If another $b_{j}=0$, then $\mathcal{L}_{j}^{\mathrm{P}, \mathrm{B}}$ is also a new constant of the motion. In this way the proper curved KC system [37] (with all the $b_{i}$ 's equal to zero) is obtained, and in that case (2.9) are just the $N$ components of the Laplace-Runge-Lenz vector on $\mathbf{S}^{N}(\kappa>0)$ and $\mathbf{H}^{N}(\kappa<0)$.

We also stress that all these examples share the same set of constants of the motion (2.2), although the geometric meaning of the canonical coordinates and momenta can be different.

\section{QMS Hamiltonians with quantum deformed $s l(2, \mathbb{R})$ coalgebra symmetry}

Here we will show that a generalization of the construction presented in the previous Section can be obtained through a quantum deformation of $s l(2, \mathbb{R})$, yielding QMS systems for certain spaces with variable curvature. Let us now state the general statement that provides a superintegrable deformation of Theorem 1.

Theorem 2. Let $\{\mathbf{q}, \mathbf{p}\}=\left\{\left(q_{1}, \ldots, q_{N}\right),\left(p_{1}, \ldots, p_{N}\right)\right\}$ be $N$ pairs of canonical variables. The ND Hamiltonian

$$
H_{z}^{(N)}=\mathcal{H}_{z}\left(\mathbf{q}^{2}, \tilde{\mathbf{p}}_{z}^{2},(\mathbf{q} \cdot \mathbf{p})_{z}\right)
$$

where $\mathcal{H}_{z}$ is any smooth function and

$$
\begin{aligned}
& \mathbf{q}^{2}=\sum_{i=1}^{N} q_{i}^{2}, \quad \tilde{\mathbf{p}}_{z}^{2}=\sum_{i=1}^{N}\left(\frac{\sinh z q_{i}^{2}}{z q_{i}^{2}} p_{i}^{2}+\frac{z b_{i}}{\sinh z q_{i}^{2}}\right) \mathrm{e}^{z K_{i}^{(N)}\left(q^{2}\right)}, \\
& (\mathbf{q} \cdot \mathbf{p})_{z}=\sum_{i=1}^{N} \frac{\sinh z q_{i}^{2}}{z q_{i}^{2}} q_{i} p_{i} \mathrm{e}^{z K_{i}^{(N)}\left(q^{2}\right)}
\end{aligned}
$$

with

$$
K_{i}^{(h)}\left(q^{2}\right)=-\sum_{k=1}^{i-1} q_{k}^{2}+\sum_{l=i+1}^{h} q_{l}^{2},
$$


is $Q M S$ for any choice of the function $\mathcal{H}$ and for arbitrary real parameters $b_{i}$. The $(2 N-3)$ functionally independent and "universal" integrals of the motion are given by

$$
\begin{aligned}
& C_{z}^{(m)}=\sum_{1 \leq i<j}^{m} Q_{i j}^{z} \mathrm{e}^{z K_{i j}^{(m)}\left(q^{2}\right)}+\sum_{i=1}^{m} b_{i} \mathrm{e}^{2 z K_{i}^{(m)}\left(q^{2}\right)}, \\
& C_{z,(m)}=\sum_{N-m+1 \leq i<j}^{N} Q_{i j}^{z} \mathrm{e}^{z \tilde{K}_{i j}^{(N-m+1)}\left(q^{2}\right)}+\sum_{i=N-m+1}^{N} b_{i} \mathrm{e}^{2 z \tilde{K}_{i}^{(N-m+1)}\left(q^{2}\right)},
\end{aligned}
$$

where $m=2, \ldots, N, C_{z}^{(N)}=C_{z,(N)}$, and

$$
\begin{aligned}
& K_{i j}^{(h)}\left(q^{2}\right)=K_{i}^{(h)}\left(q^{2}\right)+K_{j}^{(h)}\left(q^{2}\right)=-2 \sum_{k=1}^{i-1} q_{k}^{2}-q_{i}^{2}+q_{j}^{2}+2 \sum_{l=j+1}^{h} q_{l}^{2}, \\
& \tilde{K}_{i}^{(h)}\left(q^{2}\right)=-\sum_{k=h}^{i-1} q_{k}^{2}+\sum_{l=i+1}^{N} q_{l}^{2}, \\
& \tilde{K}_{i j}^{(h)}\left(q^{2}\right)=\tilde{K}_{i}^{(h)}\left(q^{2}\right)+\tilde{K}_{j}^{(h)}\left(q^{2}\right)=-2 \sum_{k=h}^{i-1} q_{k}^{2}-q_{i}^{2}+q_{j}^{2}+2 \sum_{l=j+1}^{N} q_{l}^{2}, \\
& Q_{i j}^{z}=\left\{\frac{\sinh z q_{i}^{2}}{z q_{i}^{2}} \frac{\sinh z q_{j}^{2}}{z q_{j}^{2}}\left(q_{i} p_{j}-q_{j} p_{i}\right)^{2}+\left(b_{i} \frac{\sinh z q_{j}^{2}}{\sinh z q_{i}^{2}}+b_{j} \frac{\sinh z q_{i}^{2}}{\sinh z q_{j}^{2}}\right)\right\},
\end{aligned}
$$

with $i<j$. Moreover, the sets of $N$ functions $\left\{H_{z}^{(N)}, C_{z}^{(m)}\right\}$ and $\left\{H_{z}^{(N)}, C_{z,(m)}\right\}(m=2, \ldots, N)$ are in involution.

\subsection{The proof}

The proof is based on the fact that, for any choice of the function $\mathcal{H}$, the Hamiltonian $H_{z}^{(N)}$ has a deformed Poisson coalgebra symmetry, $s l_{z}(2, \mathbb{R})$, coming (under a certain symplectic realization) from the non-standard quantum deformation of $\operatorname{sl}(2, \mathbb{R})[38,39]$ where $z$ is the deformation parameter $\left(q=\mathrm{e}^{z}\right)$. If we perform the limit $z \rightarrow 0$ in all the results given in Theorem 2 , we shall exactly recover Theorem 1 . Here we sketch the main steps of this construction, referring to $[22,35]$ for further details.

We recall that the non-standard $s l_{z}(2, \mathbb{R})$ Poisson coalgebra is given by the following deformed Poisson brackets and coproduct [22]:

$$
\begin{aligned}
& \left\{J_{3}, J_{+}\right\}=2 J_{+} \cosh z J_{-}, \quad\left\{J_{3}, J_{-}\right\}=-2 \frac{\sinh z J_{-}}{z}, \quad\left\{J_{-}, J_{+}\right\}=4 J_{3}, \\
& \Delta_{z}\left(J_{-}\right)=J_{-} \otimes 1+1, \quad \Delta_{z}\left(J_{l}\right)=J_{l} \otimes \mathrm{e}^{z J_{-}}+\mathrm{e}^{-z J_{-}} \otimes J_{l}, \quad l=+, 3 .
\end{aligned}
$$

The Casimir function for $s l_{z}(2, \mathbb{R})$ reads

$$
\mathcal{C}_{z}=\frac{\sinh z J_{-}}{z} J_{+}-J_{3}^{2} .
$$

A one-particle symplectic realization of (3.4) is given by

$$
J_{-}^{(1)}=q_{1}^{2}, \quad J_{+}^{(1)}=\frac{\sinh z q_{1}^{2}}{z q_{1}^{2}} p_{1}^{2}+\frac{z b_{1}}{\sinh z q_{1}^{2}}, \quad J_{3}^{(1)}=\frac{\sinh z q_{1}^{2}}{z q_{1}^{2}} q_{1} p_{1},
$$

where $b_{1}$ is a real parameter that labels the representation through $\mathcal{C}_{z}=b_{1}$. 
Now the essential point is the fact that the coalgebra approach [34] provides the corresponding $N$-particle symplectic realization of $s l_{z}(2, \mathbb{R})$ through the $N$-sites coproduct of (3.5) living on $\left.s l_{z}(2, \mathbb{R}) \otimes \cdots N\right) \otimes s l_{z}(2, \mathbb{R})[22]:$

$$
\begin{aligned}
& J_{-}^{(N)}=\sum_{i=1}^{N} q_{i}^{2} \equiv \mathbf{q}^{2}, \quad J_{3}^{(N)}=\sum_{i=1}^{N} \frac{\sinh z q_{i}^{2}}{z q_{i}^{2}} q_{i} p_{i} \mathrm{e}^{z K_{i}^{(N)}\left(q^{2}\right)} \equiv(\mathbf{q} \cdot \mathbf{p})_{z}, \\
& J_{+}^{(N)}=\sum_{i=1}^{N}\left(\frac{\sinh z q_{i}^{2}}{z q_{i}^{2}} p_{i}^{2}+\frac{z b_{i}}{\sinh z q_{i}^{2}}\right) \mathrm{e}^{z K_{i}^{(N)}\left(q^{2}\right)} \equiv \tilde{\mathbf{p}}_{z}^{2},
\end{aligned}
$$

where $K_{i}^{(N)}\left(q^{2}\right)$ is defined in (3.2) and $b_{i}$ are $N$ arbitrary real parameters that label the representation on each "lattice" site. This means that the $N$-particle generators (3.7) fulfil the commutation rules (3.4) with respect to the canonical Poisson bracket

$$
\{f, g\}=\sum_{i=1}^{N}\left(\frac{\partial f}{\partial q_{i}} \frac{\partial g}{\partial p_{i}}-\frac{\partial g}{\partial q_{i}} \frac{\partial f}{\partial p_{i}}\right) .
$$

Therefore the Hamiltonian (3.1) is obtained through an arbitrary smooth function $\mathcal{H}_{z}$ defined on the $N$-particle symplectic realization of the generators of $s l_{z}(2, \mathbb{R})$ :

$$
H_{z}^{(N)}=\mathcal{H}_{z}\left(J_{-}^{(N)}, J_{+}^{(N)}, J_{3}^{(N)}\right)=\mathcal{H}_{z}\left(\mathbf{q}^{2}, \tilde{\mathbf{p}}_{z}^{2},(\mathbf{q} \cdot \mathbf{p})_{z}\right) .
$$

By construction [34], the functions (3.7) Poisson commute with the $(2 N-3)$ functions (3.3) given by the sets $C_{z}^{(m)}$ and $C_{z,(m)}$, which are obtained from the "left" and "right" $m$-th coproducts of the Casimir (3.6) with $m=2,3, \ldots, N$ [35]. For instance, the $C_{z}^{(m)}$ integrals are nothing but

$$
C_{z}^{(m)}=\frac{\sinh z J_{-}^{(m)}}{z} J_{+}^{(m)}-\left(J_{3}^{(m)}\right)^{2},
$$

and the right ones $C_{z,(m)}$ can be obtained through an appropriate permutation of the labelling of the lattice sites (note that these integrals depend on the canonical coordinates running from $(N-m+1)$ up to $N)$. Thus $H_{z}^{(N)}$ Poisson commutes with the $(2 N-3)$ integrals and, furthermore, the coalgebra symmetry also ensures that each of the subsets $\left\{C_{z}^{(2)}, \ldots, C_{z}^{(N)}, H_{z}^{(N)}\right\}$ and $\left\{C_{z,(2)}, \ldots, C_{z,(N)}, H_{z}^{(N)}\right\}$ consists of $N$ functions in involution.

In order to prove the functional independence of the $2 N-2$ functions $\left\{C_{z}^{(2)}, C_{z}^{(3)}, \ldots, C_{z}^{(N)} \equiv\right.$ $\left.C_{z,(N)}, C_{z,(N-1)}, \ldots, C_{z,(2)}, H_{z}^{(N)}\right\}$ it suffices to realize that such functions are just deformations in the deformation parameter $z$ of the $\operatorname{sl}(2, \mathbb{R})$ integrals given by $(2.2)$, and the latter (which are recovered when $z \rightarrow 0$ ) are indeed functionally independent.

Thus, we conclude that any arbitrary function $\mathcal{H}_{z}$ (3.8) defines a QMS Hamiltonian system.

\subsection{The $N=2$ case}

In order to illustrate the previous construction, let us explicitly write the 2-particle symplectic realization of $s l_{z}(2, \mathbb{R})(3.7)$ :

$$
\begin{aligned}
& J_{-}^{(2)}=q_{1}^{2}+q_{2}^{2}, \quad J_{3}^{(2)}=\frac{\sinh z q_{1}^{2}}{z q_{1}^{2}} \mathrm{e}^{z q_{2}^{2}} q_{1} p_{1}+\frac{\sinh z q_{2}^{2}}{z q_{2}^{2}} \mathrm{e}^{-z q_{1}^{2}} q_{2} p_{2}, \\
& J_{+}^{(2)}=\frac{\sinh z q_{1}^{2}}{z q_{1}^{2}} \mathrm{e}^{z q_{2}^{2}} p_{1}^{2}+\frac{\sinh z q_{2}^{2}}{z q_{2}^{2}} \mathrm{e}^{-z q_{1}^{2}} p_{2}^{2}+\frac{z b_{1}}{\sinh z q_{1}^{2}} \mathrm{e}^{z q_{2}^{2}}+\frac{z b_{2}}{\sinh z q_{2}^{2}} \mathrm{e}^{-z q_{1}^{2}}
\end{aligned}
$$


In this case there is a single (left and right) constant of the motion:

$$
C_{z}^{(2)}=\frac{\sinh z J_{-}^{(2)}}{z} J_{+}^{(2)}-\left(J_{3}^{(2)}\right)^{2}
$$

After some straightforward computations this integral can be expressed as

$$
\begin{aligned}
C_{z}^{(2)}= & \frac{\sinh z q_{1}^{2}}{z q_{1}^{2}} \frac{\sinh z q_{2}^{2}}{z q_{2}^{2}}\left(q_{1} p_{2}-q_{2} p_{1}\right)^{2} \mathrm{e}^{z\left(q_{2}^{2}-q_{1}^{2}\right)}+b_{1} \mathrm{e}^{2 z q_{2}^{2}}+b_{2} \mathrm{e}^{-2 z q_{1}^{2}} \\
& +\left(b_{1} \frac{\sinh z q_{2}^{2}}{\sinh z q_{1}^{2}}+b_{2} \frac{\sinh z q_{1}^{2}}{\sinh z q_{2}^{2}}\right) \mathrm{e}^{z\left(q_{2}^{2}-q_{1}^{2}\right)}
\end{aligned}
$$

By construction, this constant of the motion will Poisson-commute with all the Hamiltonians

$$
H_{z}^{(2)}=\mathcal{H}_{z}\left(J_{-}^{(2)}, J_{+}^{(2)}, J_{3}^{(2)}\right) .
$$

Note that in the $N=2$ case quasi-maximal superintegrability means only integrability, i.e., the only constant given by Theorem 2 is just $C_{z}^{(2)} \equiv C_{z,(2)}$; this fact does not exclude that there could be some specific choices for $\mathcal{H}_{z}$ for which an additional integral does exist. When $N \geq 3$, Theorem 2 will always provide QMS Hamiltonians.

\section{Free motion on $2 \mathrm{D}$ and $3 \mathrm{D}$ curved manifolds}

\subsection{D curved manifolds}

Throughout this Section we will consider only free motion. Therefore we shall take the symplectic realization with $b_{1}=b_{2}=0$ in order to avoid centrifugal potential terms. In general, we can consider an infinite family of integrable (and quadratic in the momenta) free $N=2$ motions with $s l_{z}(2, \mathbb{R})$ coalgebra symmetry through Hamiltonians of the type

$$
H_{z}^{(2)}=\frac{1}{2} J_{+}^{(2)} f\left(z J_{-}^{(2)}\right)
$$

where $f$ is an arbitrary smooth function such that $\lim _{z \rightarrow 0} f\left(z J_{-}^{(2)}\right)=1$, that is, $\lim _{z \rightarrow 0} H_{z}^{(2)}=\frac{1}{2}\left(p_{1}^{2}+\right.$ $p_{2}^{2}$ ). We shall explore in the sequel some specific choices for $f$, and we shall analyse the spaces generated by them.

\subsubsection{An integrable case}

Of course, the simplest choice will be just to set $f \equiv 1[31]$ :

$$
\mathcal{H}_{z}^{\mathrm{I}}=\frac{1}{2} J_{+}^{(2)}=\frac{1}{2}\left(\frac{\sinh z q_{1}^{2}}{z q_{1}^{2}} \mathrm{e}^{z q_{2}^{2}} p_{1}^{2}+\frac{\sinh z q_{2}^{2}}{z q_{2}^{2}} \mathrm{e}^{-z q_{1}^{2}} p_{2}^{2}\right) .
$$

Hence the kinetic energy $\mathcal{T}_{z}^{\mathrm{I}}\left(q_{i}, p_{i}\right)$ coming from $\mathcal{H}_{z}^{\mathrm{I}}$ is

$$
\mathcal{T}_{z}^{\mathrm{I}}\left(q_{i}, \dot{q}_{i}\right)=\frac{1}{2}\left(\frac{z q_{1}^{2}}{\sinh z q_{1}^{2}} \mathrm{e}^{-z q_{2}^{2}} \dot{q}_{1}^{2}+\frac{z q_{2}^{2}}{\sinh z q_{2}^{2}} \mathrm{e}^{z q_{1}^{2}} \dot{q}_{2}^{2}\right),
$$

and defines a geodesic flow on a 2D Riemannian space with signature diag $(+,+)$ and metric given by:

$$
\mathrm{d} s_{I}^{2}=\frac{2 z q_{1}^{2}}{\sinh z q_{1}^{2}} \mathrm{e}^{-z q_{2}^{2}} \mathrm{~d} q_{1}^{2}+\frac{2 z q_{2}^{2}}{\sinh z q_{2}^{2}} \mathrm{e}^{z q_{1}^{2}} \mathrm{~d} q_{2}^{2} .
$$


The Gaussian curvature $K$ for this space can be computed through

$$
K=\frac{-1}{\sqrt{g_{11} g_{22}}}\left\{\frac{\partial}{\partial q_{1}}\left(\frac{1}{\sqrt{g_{11}}} \frac{\partial \sqrt{g_{22}}}{\partial q_{1}}\right)+\frac{\partial}{\partial q_{2}}\left(\frac{1}{\sqrt{g_{22}}} \frac{\partial \sqrt{g_{11}}}{\partial q_{2}}\right)\right\}
$$

and turns out to be non-constant and negative:

$$
K\left(q_{1}, q_{2} ; z\right)=-z \sinh \left(z\left(q_{1}^{2}+q_{2}^{2}\right)\right) .
$$

Therefore, the underlying 2D space is of hyperbolic type and endowed with a "radial" symmetry.

Let us now consider the following change of coordinates that includes a new parameter $\lambda_{2} \neq 0$ :

$$
\cosh \left(\lambda_{1} \rho\right)=\exp \left\{z\left(q_{1}^{2}+q_{2}^{2}\right)\right\}, \quad \sin ^{2}\left(\lambda_{2} \theta\right)=\frac{\exp \left\{2 z q_{1}^{2}\right\}-1}{\exp \left\{2 z\left(q_{1}^{2}+q_{2}^{2}\right)\right\}-1},
$$

where $z=\lambda_{1}^{2}$ and $\lambda_{2}$ can take either a real or a pure imaginary value. Note that the new variable $\cosh \left(\lambda_{1} \rho\right)$ is a collective variable, a function of $\Delta\left(J_{-}\right)$; its role will be specified later. On the other hand, the zero-deformation limit $z \rightarrow 0$ is in fact the flat limit $K \rightarrow 0$, since in this limit

$$
\rho \rightarrow 2\left(q_{1}^{2}+q_{2}^{2}\right), \quad \sin ^{2}\left(\lambda_{2} \theta\right) \rightarrow \frac{q_{1}^{2}}{q_{1}^{2}+q_{2}^{2}}
$$

Thus $\rho$ can be interpreted as a radial coordinate and $\theta$ is either a circular $\left(\lambda_{2}\right.$ real $)$ or a hyperbolic angle ( $\lambda_{2}$ imaginary). Notice that in the latter case, say $\lambda_{2}=\mathrm{i}$, the coordinate $q_{1}$ is imaginary and can be written as $q_{1}=\mathrm{i} \tilde{q}_{1}$ where $\tilde{q}_{1}$ is a real coordinate; then $\rho \rightarrow 2\left(q_{2}^{2}-\tilde{q}_{1}^{2}\right)$ which corresponds to a relativistic radial distance. Therefore the introduction of the additional parameter $\lambda_{2}$ will allow us to obtain Lorentzian metrics.

In this new coordinates, the metric (4.4) reads

$$
\mathrm{d} s_{I}^{2}=\frac{1}{\cosh \left(\lambda_{1} \rho\right)}\left(\mathrm{d} \rho^{2}+\lambda_{2}^{2} \frac{\sinh ^{2}\left(\lambda_{1} \rho\right)}{\lambda_{1}^{2}} \mathrm{~d} \theta^{2}\right)=\frac{1}{\cosh \left(\lambda_{1} \rho\right)} \mathrm{d} s_{0}^{2},
$$

where $\mathrm{d} s_{0}^{2}$ is just the metric of the $2 \mathrm{D}$ Cayley-Klein spaces in terms of geodesic polar coordinates $[40,41]$ provided that we identify $z=\lambda_{1}^{2} \equiv-\kappa_{1}$ and $\lambda_{2}^{2} \equiv \kappa_{2}$; hence $\lambda_{2}$ determines the signature of the metric. The Gaussian curvature turns out to be

$$
K(\rho)=-\frac{1}{2} \lambda_{1}^{2} \frac{\sinh ^{2}\left(\lambda_{1} \rho\right)}{\cosh \left(\lambda_{1} \rho\right)}
$$

In this way we find the following spaces, whose main properties are summarized in Table 1:

- When $\lambda_{2}$ is real, we get a $2 \mathrm{D}$ deformed sphere $\mathbf{S}_{z}^{2}(z<0)$, and a deformed hyperbolic or Lobachewski space $\mathbf{H}_{z}^{2}(z>0)$.

- When $\lambda_{2}$ is imaginary, we obtain a deformation of the $(1+1) \mathrm{D}$ anti-de Sitter spacetime $\mathbf{A d S}_{z}^{1+1}(z<0)$ and of the de Sitter one $\mathbf{d S}_{z}^{1+1}(z>0)$.

- In the non-deformed case $z \rightarrow 0$, the Euclidean space $\mathbf{E}^{2}$ ( $\lambda_{2}$ real) and Minkowskian spacetime $\mathbf{M}^{1+1}$ ( $\lambda_{2}$ imaginary) are recovered.

Accordingly, the kinetic energy (4.3) is transformed into

$$
\mathcal{T}_{z}^{\mathrm{I}}(\rho, \theta ; \dot{\rho}, \dot{\theta})=\frac{1}{2 \cosh \left(\lambda_{1} \rho\right)}\left(\dot{\rho}^{2}+\lambda_{2}^{2} \frac{\sinh ^{2}\left(\lambda_{1} \rho\right)}{\lambda_{1}^{2}} \dot{\theta}^{2}\right),
$$


Table 1. Metric and Gaussian curvature of the $2 \mathrm{D}$ spaces with $s_{z}(2, \mathbb{R})$ coalgebra symmetry for different values of the deformation parameter $z=\lambda_{1}^{2}$ and signature parameter $\lambda_{2}$.

\begin{tabular}{ll}
\hline 2 D deformed Riemannian spaces & $(1+1)$ D deformed relativistic spacetimes \\
\hline$\bullet$ Deformed sphere $\mathbf{S}_{z}^{2}$ & $\bullet$ Deformed anti-de Sitter spacetime $\mathbf{A d S}_{z}^{1+1}$ \\
$z=-1 ;\left(\lambda_{1}, \lambda_{2}\right)=(\mathrm{i}, 1)$ & $z=-1 ;\left(\lambda_{1}, \lambda_{2}\right)=(\mathrm{i}, \mathrm{i})$ \\
$\mathrm{d} s^{2}=\frac{1}{\cos \rho}\left(\mathrm{d} \rho^{2}+\sin ^{2} \rho \mathrm{d} \theta^{2}\right)$ & $\mathrm{d} s^{2}=\frac{1}{\cos \rho}\left(\mathrm{d} \rho^{2}-\sin ^{2} \rho \mathrm{d} \theta^{2}\right)$ \\
$K=-\frac{\sin ^{2} \rho}{2 \cos \rho}$ & $K=-\frac{\sin ^{2} \rho}{2 \cos \rho}$ \\
$\bullet$ Euclidean space $\mathbf{E}^{2}$ & $\bullet$ Minkowskian spacetime $\mathbf{M}^{1+1}$ \\
$z=0 ;\left(\lambda_{1}, \lambda_{2}\right)=(0,1)$ & $z=0 ;\left(\lambda_{1}, \lambda_{2}\right)=(0, \mathrm{i})$ \\
$\mathrm{d} s^{2}=\mathrm{d} \rho^{2}+\rho^{2} \mathrm{~d} \theta^{2}$ & $\mathrm{~d} s^{2}=\mathrm{d} \rho^{2}-\rho^{2} \mathrm{~d} \theta^{2}$ \\
$K=0$ & $K=0$ \\
$\bullet$ Deformed hyperbolic space $\mathbf{H}_{z}^{2}$ & $\bullet$ Deformed $\mathrm{de} \operatorname{Sitter} \operatorname{spacetime} \mathbf{d} \mathbf{S}_{z}^{1+1}$ \\
$z=1 ;\left(\lambda_{1}, \lambda_{2}\right)=(1,1)$ & $z=1 ;\left(\lambda_{1}, \lambda_{2}\right)=(1, \mathrm{i})$ \\
$\mathrm{d} s^{2}=\frac{1}{\cosh \rho}\left(\mathrm{d} \rho^{2}+\sinh ^{2} \rho \mathrm{d} \theta^{2}\right)$ & $\mathrm{d} s^{2}=\frac{1}{\cosh \rho}\left(\mathrm{d} \rho^{2}-\sinh ^{2} \rho \mathrm{d} \theta^{2}\right)$ \\
$K=-\frac{\sinh { }^{2} \rho}{2 \cosh \rho}$ & $K=-\frac{\sinh { }^{2} \rho}{2 \cosh \rho}$ \\
\hline
\end{tabular}

and the free motion Hamiltonian (4.2) is written as

$$
\widetilde{H}_{z}^{\mathrm{I}}=\frac{1}{2} \cosh \left(\lambda_{1} \rho\right)\left(p_{\rho}^{2}+\frac{\lambda_{1}^{2}}{\lambda_{2}^{2} \sinh ^{2}\left(\lambda_{1} \rho\right)} p_{\theta}^{2}\right)
$$

where $\widetilde{H}_{z}^{\mathrm{I}}=2 \mathcal{H}_{z}^{\mathrm{I}}$. There is a unique constant of the motion $C_{z}^{(2)} \equiv C_{z,(2)}(3.9)$ which in terms of the new phase space is simply given by

$$
\widetilde{C}_{z}=p_{\theta}^{2},
$$

provided that $\widetilde{C}_{z}=4 \lambda_{2}^{2} C_{z}^{(2)}$. This allows us to apply a radial-symmetry reduction:

$$
\widetilde{H}_{z}^{\mathrm{I}}=\frac{1}{2} \cosh \left(\lambda_{1} \rho\right) p_{\rho}^{2}+\frac{\lambda_{1}^{2} \cosh \left(\lambda_{1} \rho\right)}{2 \lambda_{2}^{2} \sinh ^{2}\left(\lambda_{1} \rho\right)} \widetilde{C}_{z}
$$

We remark that the explicit integration of the geodesic motion on all these spaces can be explicitly performed in terms of elliptic integrals.

\subsubsection{The superintegrable case}

A MS Hamiltonian is given by

$$
\mathcal{H}_{z}^{\mathrm{MS}}=\frac{1}{2} J_{+}^{(2)} \mathrm{e}^{z J_{-}^{(2)}}=\frac{1}{2}\left(\frac{\sinh z q_{1}^{2}}{z q_{1}^{2}} \mathrm{e}^{z q_{1}^{2}} \mathrm{e}^{2 z q_{2}^{2}} p_{1}^{2}+\frac{\sinh z q_{2}^{2}}{z q_{2}^{2}} \mathrm{e}^{z q_{2}^{2}} p_{2}^{2}\right),
$$

since there exists an additional (and functionally independent) constant of the motion [22]:

$$
\mathcal{I}_{z}=\frac{\sinh z q_{1}^{2}}{2 z q_{1}^{2}} \mathrm{e}^{z q_{1}^{2}} p_{1}^{2}
$$


This choice corresponds to the kinetic energy

$$
\mathcal{T}_{z}^{\mathrm{MS}}\left(q_{i}, \dot{q}_{i}\right)=\frac{1}{2}\left(\frac{z q_{1}^{2}}{\sinh z q_{1}^{2}} \mathrm{e}^{-z q_{1}^{2}} \mathrm{e}^{-2 z q_{2}^{2}} \dot{q}_{1}^{2}+\frac{z q_{2}^{2}}{\sinh z q_{2}^{2}} \mathrm{e}^{-z q_{2}^{2}} \dot{q}_{2}^{2}\right),
$$

whose associated metric is

$$
\mathrm{d} s_{\mathrm{MS}}^{2}=\frac{2 z q_{1}^{2}}{\sinh z q_{1}^{2}} \mathrm{e}^{-z q_{1}^{2}} \mathrm{e}^{-2 z q_{2}^{2}} \mathrm{~d} q_{1}^{2}+\frac{2 z q_{2}^{2}}{\sinh z q_{2}^{2}} \mathrm{e}^{-z q_{2}^{2}} \mathrm{~d} q_{2}^{2} .
$$

Surprisingly enough, the computation of the Gaussian curvature $K$ for $\mathrm{d} s_{\text {MS }}^{2}$ gives that $K=z$. Therefore, we are dealing with a space of constant curvature which is just the deformation parameter $z$. In [31] it was shown that a certain change of coordinates (that includes the signature parameter $\lambda_{2}$ ) transforms the metric into

$$
\mathrm{d} s_{\mathrm{MS}}^{2}=\mathrm{d} r^{2}+\lambda_{2}^{2} \frac{\sin ^{2}\left(\lambda_{1} r\right)}{\lambda_{1}^{2}} \mathrm{~d} \theta^{2},
$$

which exactly coincides with the metric of the Cayley-Klein spaces written in geodesic polar coordinates $(r, \theta)$ provided that now $z=\lambda_{1}^{2} \equiv \kappa_{1}$ and $\lambda_{2}^{2} \equiv \kappa_{2}$. Obviously, after this change of variables the geodesic motion can be reduced to a "radial" 1D system:

$$
\widetilde{H}_{z}^{\mathrm{MS}}=\frac{1}{2} p_{r}^{2}+\frac{\lambda_{1}^{2}}{2 \lambda_{2}^{2} \sin ^{2}\left(\lambda_{1} r\right)} \widetilde{C}_{z}
$$

where $\widetilde{H}_{z}^{\mathrm{MS}}=2 \mathcal{H}_{z}^{\mathrm{MS}}$ and $\widetilde{C}_{z}=p_{\theta}^{2}$ is, as in the previous case, the usual generalized momentum for the $\theta$ coordinate.

\subsubsection{A more general case}

At this point, one could wonder whether there exist other choices for the Hamiltonian yielding constant curvature. In fact, let us consider the generic Hamiltonian (4.1) depending on $f$. If we compute the general expression for the 2D Gaussian curvature in terms of the function $f(x)$ we find that

$$
K(x)=z\left(f^{\prime}(x) \cosh x+\left(f^{\prime \prime}(x)-f(x)-\frac{f^{\prime 2}(x)}{f(x)}\right) \sinh x\right),
$$

where $x \equiv z J_{-}=z\left(q_{1}^{2}+q_{2}^{2}\right), f^{\prime}=\frac{\mathrm{d} f(x)}{\mathrm{d} x}$ and $f^{\prime \prime}=\frac{\mathrm{d}^{2} f(x)}{\mathrm{d} x^{2}}$. Thus, in general, we obtain spaces with variable curvature. In order to characterize the constant curvature cases, we can define $g:=f^{\prime} / f$ and write

$$
K / z=f^{\prime} \cosh x+\left(f^{\prime \prime}-f-\left(f^{\prime}\right)^{2} / f\right) \sinh x=f\left(g \cosh x+\left(g^{\prime}-1\right) \sinh x\right) .
$$

If we now require $K$ to be a constant we get the equation

$$
K^{\prime}=0 \equiv 2 y \cosh x+y^{\prime} \sinh x=0, \quad \text { where } \quad y:=2 g^{\prime}+g^{2}-1 .
$$

The solution for this equation yields

$$
y=\frac{A}{\sinh ^{2} x},
$$

where $A$ is a constant, and solving for $g$, we get for $F:=f^{\frac{1}{2}}$ the equation

$$
F^{\prime \prime}=\frac{1}{4}\left(1+\frac{A}{\sinh ^{2} x}\right) F,
$$


whose general solution is $(A:=\lambda(\lambda-1))$ :

$$
F=(\sinh x)^{\lambda}\left\{C_{1}(\sinh (x / 2))^{(1-2 \lambda)}+C_{2}(\cosh (x / 2))^{(1-2 \lambda)}\right\},
$$

where $C_{1}$ and $C_{2}$ are two integration constants.

Therefore, many different solutions lead to $2 \mathrm{D}$ constant curvature spaces. However, we must impose as additional condition that $\lim _{x \rightarrow 0} f=1$. In this way we obtain that only the cases with $A=0$ are possible, that is, either $\lambda \stackrel{x \rightarrow 0}{=}$ or $\lambda=0$. Hence the two elementary solutions are just the Hamiltonians

$$
\mathcal{H}_{z}=\frac{1}{2} J_{+} \mathrm{e}^{ \pm z J_{-}}
$$

and the curvature of their associated spaces is $K= \pm z$.

\subsection{D curved manifolds}

The study of the 3D case follows exactly the same pattern. The three-particle symplectic realization of $s l_{z}(2, \mathbb{R})$ (with all $b_{i}=0$ ) is obtained from (3.7):

$$
\begin{aligned}
& J_{-}^{(3)}=q_{1}^{2}+q_{2}^{2}+q_{3}^{2} \equiv \mathbf{q}^{2}, \\
& J_{+}^{(3)}=\frac{\sinh z q_{1}^{2}}{z q_{1}^{2}} p_{1}^{2} \mathrm{e}^{z q_{2}^{2}} \mathrm{e}^{z q_{3}^{2}}+\frac{\sinh z q_{2}^{2}}{z q_{2}^{2}} p_{2}^{2} \mathrm{e}^{-z q_{1}^{2}} \mathrm{e}^{z q_{3}^{2}}+\frac{\sinh z q_{3}^{2}}{z q_{3}^{2}} p_{3}^{2} \mathrm{e}^{-z q_{1}^{2}} \mathrm{e}^{-z q_{2}^{2}}, \\
& J_{3}^{(3)}=\frac{\sinh z q_{1}^{2}}{z q_{1}^{2}} q_{1} p_{1} \mathrm{e}^{z q_{2}^{2}} \mathrm{e}^{z q_{3}^{2}}+\frac{\sinh z q_{2}^{2}}{z q_{2}^{2}} q_{2} p_{2} \mathrm{e}^{-z q_{1}^{2}} \mathrm{e}^{z q_{3}^{2}}+\frac{\sinh z q_{3}^{2}}{z q_{3}^{2}} q_{3} p_{3} \mathrm{e}^{-z q_{1}^{2}} \mathrm{e}^{-z q_{2}^{2}} .
\end{aligned}
$$

By construction, these generators Poisson-commute with the three integrals $\left\{C_{z}^{(2)}, C_{z}^{(3)} \equiv C_{z,(3)}\right.$, $\left.C_{z,(2)}\right\}$ given in $(3.3)$ :

$$
\begin{aligned}
C_{z}^{(2)}= & \frac{\sinh z q_{1}^{2}}{z q_{1}^{2}} \frac{\sinh z q_{2}^{2}}{z q_{2}^{2}}\left(q_{1} p_{2}-q_{2} p_{1}\right)^{2} \mathrm{e}^{-z q_{1}^{2}} \mathrm{e}^{z q_{2}^{2}}, \\
C_{z,(2)}= & \frac{\sinh z q_{2}^{2}}{z q_{2}^{2}} \frac{\sinh z q_{3}^{2}}{z q_{3}^{2}}\left(q_{2} p_{3}-q_{3} p_{2}\right)^{2} \mathrm{e}^{-z q_{2}^{2}} \mathrm{e}^{z q_{3}^{2}}, \\
C_{z}^{(3)}= & \frac{\sinh z q_{1}^{2}}{z q_{1}^{2}} \frac{\sinh z q_{2}^{2}}{z q_{2}^{2}}\left(q_{1} p_{2}-q_{2} p_{1}\right)^{2} \mathrm{e}^{-z q_{1}^{2}} \mathrm{e}^{z q_{2}^{2}} \mathrm{e}^{2 z q_{3}^{2}} \\
& +\frac{\sinh z q_{1}^{2}}{z q_{1}^{2}} \frac{\sinh z q_{3}^{2}}{z q_{3}^{2}}\left(q_{1} p_{3}-q_{3} p_{1}\right)^{2} \mathrm{e}^{-z q_{1}^{2}} \mathrm{e}^{z q_{3}^{2}} \\
& +\frac{\sinh z q_{2}^{2}}{z q_{2}^{2}} \frac{\sinh z q_{3}^{2}}{z q_{3}^{2}}\left(q_{2} p_{3}-q_{3} p_{2}\right)^{2} \mathrm{e}^{-2 z q_{1}^{2}} \mathrm{e}^{-z q_{2}^{2}} \mathrm{e}^{z q_{3}^{2}} .
\end{aligned}
$$

\subsubsection{QMS free motion: non-constant curvature}

If we now consider the kinetic energy $\mathcal{T}_{z}\left(q_{i}, \dot{q}_{i}\right)$ coming from the Hamiltonian

$$
\mathcal{H}_{z}\left(q_{i}, p_{i}\right)=\frac{1}{2} J_{+}^{(3)}
$$

it corresponds to the free Lagrangian [33]

$$
\mathcal{T}_{z}=\frac{1}{2}\left(\frac{z q_{1}^{2}}{\sinh z q_{1}^{2}} \mathrm{e}^{-z q_{2}^{2}} \mathrm{e}^{-z q_{3}^{2}} \dot{q}_{1}^{2}+\frac{z q_{2}^{2}}{\sinh z q_{2}^{2}} \mathrm{e}^{z q_{1}^{2}} \mathrm{e}^{-z q_{3}^{2}} \dot{q}_{2}^{2}+\frac{z q_{3}^{2}}{\sinh z q_{3}^{2}} \mathrm{e}^{z q_{1}^{2}} \mathrm{e}^{z q_{2}^{2}} \dot{q}_{3}^{2}\right),
$$


that defines a geodesic flow on a 3D Riemannian space with metric

$$
\mathrm{d} s^{2}=\frac{2 z q_{1}^{2}}{\sinh z q_{1}^{2}} \mathrm{e}^{-z q_{2}^{2}} \mathrm{e}^{-z q_{3}^{2}} \mathrm{~d} q_{1}^{2}+\frac{2 z q_{2}^{2}}{\sinh z q_{2}^{2}} \mathrm{e}^{z q_{1}^{2}} \mathrm{e}^{-z q_{3}^{2}} \mathrm{~d} q_{2}^{2}+\frac{2 z q_{3}^{2}}{\sinh z q_{3}^{2}} \mathrm{e}^{z q_{1}^{2}} \mathrm{e}^{z q_{2}^{2}} \mathrm{~d} q_{3}^{2} .
$$

The corresponding sectional curvatures $K_{i j}$ are

$$
\begin{aligned}
& K_{12}=\frac{z}{4} \mathrm{e}^{-z \mathbf{q}^{2}}\left(1+\mathrm{e}^{2 z q_{3}^{2}}-2 \mathrm{e}^{2 z \mathbf{q}^{2}}\right), \\
& K_{13}=\frac{z}{4} \mathrm{e}^{-z \mathbf{q}^{2}}\left(2-\mathrm{e}^{2 z q_{3}^{2}}+\mathrm{e}^{2 z q_{2}^{2}} \mathrm{e}^{2 z q_{3}^{2}}-2 \mathrm{e}^{2 z \mathbf{q}^{2}}\right), \\
& K_{23}=\frac{z}{4} \mathrm{e}^{-z \mathbf{q}^{2}}\left(2-\mathrm{e}^{2 z q_{2}^{2}} \mathrm{e}^{2 z q_{3}^{2}}-2 \mathrm{e}^{2 z \mathbf{q}^{2}}\right) .
\end{aligned}
$$

The following nice expression for the scalar curvature $K$ is found:

$$
K_{12}+K_{13}+K_{23}=-\frac{5}{2} z \sinh \left(z \mathbf{q}^{2}\right)=K / 2 .
$$

Once again, the radial symmetry can be explicitly emphasized through new canonical coordinates $(\rho, \theta, \phi)$ defined by:

$$
\begin{aligned}
& \cosh ^{2}\left(\lambda_{1} \rho\right)=\mathrm{e}^{2 z \mathbf{q}^{2}}, \\
& \sinh ^{2}\left(\lambda_{1} \rho\right) \cos ^{2}\left(\lambda_{2} \theta\right)=\mathrm{e}^{2 z q_{1}^{2}} \mathrm{e}^{2 z q_{2}^{2}}\left(\mathrm{e}^{2 z q_{3}^{2}}-1\right), \\
& \sinh ^{2}\left(\lambda_{1} \rho\right) \sin ^{2}\left(\lambda_{2} \theta\right) \cos ^{2} \phi=\mathrm{e}^{2 z q_{1}^{2}}\left(\mathrm{e}^{2 z q_{2}^{2}}-1\right), \\
& \sinh ^{2}\left(\lambda_{1} \rho\right) \sin ^{2}\left(\lambda_{2} \theta\right) \sin ^{2} \phi=\mathrm{e}^{2 z q_{1}^{2}}-1,
\end{aligned}
$$

where $z=\lambda_{1}^{2}$ and $\lambda_{2} \neq 0$ is the additional signature parameter, that will allow for the presence of relativistic spaces. Under this change of variables, the metric is transformed into

$$
\mathrm{d} s^{2}=\frac{1}{\cosh \left(\lambda_{1} \rho\right)}\left(\mathrm{d} \rho^{2}+\lambda_{2}^{2} \frac{\sinh ^{2}\left(\lambda_{1} \rho\right)}{\lambda_{1}^{2}}\left(\mathrm{~d} \theta^{2}+\frac{\sinh ^{2}\left(\lambda_{2} \theta\right)}{\lambda_{2}^{2}} \mathrm{~d} \phi^{2}\right)\right) .
$$

This is just the metric of the 3D Riemannian and relativistic spacetimes written in geodesic polar coordinates and multiplied by a global factor $1 / \cosh \left(\lambda_{1} \rho\right)$ that encodes the information concerning the variable curvature of the space.

Sectional and scalar curvatures are now written in the form

$$
K_{12}=K_{13}=-\frac{1}{2} \lambda_{1}^{2} \frac{\sinh ^{2}\left(\lambda_{1} \rho\right)}{\cosh \left(\lambda_{1} \rho\right)}, \quad K_{23}=K_{12} / 2, \quad K=-\frac{5}{2} \lambda_{1}^{2} \frac{\sinh ^{2}\left(\lambda_{1} \rho\right)}{\cosh \left(\lambda_{1} \rho\right)} .
$$

Therefore, according to the values of $\left(\lambda_{1}, \lambda_{2}\right)$ we have obtained a deformation of the 3D sphere $(i, 1)$, hyperbolic $(1,1)$, de Sitter $(1, i)$ and anti-de Sitter $(i, i)$ spaces. The "classical" limit $z \rightarrow 0$ corresponds to a zero-curvature limit leading to the proper Euclidean $(0,1)$ and Minkowskian $(0, i)$ spaces.

The QMS Hamiltonian (4.7), that determines the free motion on the above spaces, and its three integrals of the motion (4.6) are written in terms of the new canonical coordinates $(\rho, \theta, \phi)$ and conjugated momenta $\left(p_{\rho}, p_{\theta}, p_{\phi}\right)$ as

$$
\begin{aligned}
& \tilde{H}_{z}=\frac{1}{2} \cosh \left(\lambda_{1} \rho\right)\left(p_{\rho}^{2}+\frac{\lambda_{1}^{2}}{\lambda_{2}^{2} \sinh ^{2}\left(\lambda_{1} \rho\right)}\left(p_{\theta}^{2}+\frac{\lambda_{2}^{2}}{\sin ^{2}\left(\lambda_{2} \theta\right)} p_{\phi}^{2}\right)\right), \\
& \tilde{C}_{z}^{(2)}=p_{\phi}^{2}, \quad \tilde{C}_{z}^{(3)}=p_{\theta}^{2}+\frac{\lambda_{2}^{2}}{\sin ^{2}\left(\lambda_{2} \theta\right)} p_{\phi}^{2}, \quad \tilde{C}_{z,(2)}=\left(\cos \phi p_{\theta}-\lambda_{2} \frac{\sin \phi p_{\phi}}{\tan \left(\lambda_{2} \theta\right)}\right)^{2},
\end{aligned}
$$

provided that $\tilde{H}_{z}=2 \mathcal{H}_{z}, \tilde{C}_{z}^{(2)}=4 C_{z}^{(2)}, \tilde{C}_{z,(2)}=4 \lambda_{2}^{2} C_{z,(2)}$ and $\tilde{C}_{z}^{(3)}=4 \lambda_{2}^{2} C_{z}^{(3)}$. 
Furthermore the set of three functions $\left\{\tilde{C}_{z}^{(2)}, \tilde{C}_{z}^{(3)}, \tilde{H}_{z}\right\}$, which characterizes the complete integrability of the Hamiltonian, allows us to write three equations, each of them depending on a canonical pair:

$$
\begin{aligned}
& \tilde{C}_{z}^{(2)}\left(\phi, p_{\phi}\right)=p_{\phi}^{2}, \quad \tilde{C}_{z}^{(3)}\left(\theta, p_{\theta}\right)=p_{\theta}^{2}+\frac{\lambda_{2}^{2}}{\sin ^{2}\left(\lambda_{2} \theta\right)} \tilde{C}_{z}^{(2)}, \\
& \tilde{H}_{z}\left(\rho, p_{\rho}\right)=\frac{1}{2} \cosh \left(\lambda_{1} \rho\right)\left(p_{\rho}^{2}+\frac{\lambda_{1}^{2}}{\lambda_{2}^{2} \sinh ^{2}\left(\lambda_{1} \rho\right)} \tilde{C}_{z}^{(3)}\right) .
\end{aligned}
$$

Therefore the Hamiltonian is separable and reduced to a 1D radial system.

\subsubsection{MS free motion: constant curvature}

The following choice for the Hamiltonian

$$
\mathcal{H}_{z}^{\mathrm{MS}}=\frac{1}{2} J_{+}^{(3)} \mathrm{e}^{z J_{-}^{(3)},}
$$

yields a MS system since it has four (functionally independent) constants of motion, the three universal integrals (4.6) together with $\mathcal{I}_{z}$ (4.5). In fact, this the $3 \mathrm{D}$ version of the Hamiltonian described in Section 4.1.2.

The associated kinetic energy is

$$
\mathcal{T}_{z}^{\mathrm{MS}}=\frac{1}{2}\left(\frac{z q_{1}^{2}}{\sinh z q_{1}^{2}} \mathrm{e}^{z q_{1}^{2}} \dot{q}_{1}^{2}+\frac{z q_{2}^{2}}{\sinh z q_{2}^{2}} \mathrm{e}^{2 z q_{1}^{2}} \mathrm{e}^{z q_{2}^{2}} \dot{q}_{2}^{2}+\frac{z q_{3}^{2}}{\sinh z q_{3}^{2}} \mathrm{e}^{2 z q_{1}^{2}} \mathrm{e}^{2 z q_{2}^{2}} \mathrm{e}^{z q_{3}^{2}} \dot{q}_{3}^{2}\right),
$$

and the underlying metric reads

$$
\mathrm{d} s_{\mathrm{MS}}^{2}=\frac{2 z q_{1}^{2}}{\sinh z q_{1}^{2}} \mathrm{e}^{z q_{1}^{2}} \mathrm{~d} q_{1}^{2}+\frac{2 z q_{2}^{2}}{\sinh z q_{2}^{2}} \mathrm{e}^{2 z q_{1}^{2}} \mathrm{e}^{z q_{2}^{2}} \mathrm{~d} q_{2}^{2}+\frac{2 z q_{3}^{2}}{\sinh z q_{3}^{2}} \mathrm{e}^{2 z q_{1}^{2}} \mathrm{e}^{2 z q_{2}^{2}} \mathrm{e}^{z q_{3}^{2}} \mathrm{~d} q_{3}^{2} .
$$

This space is again a Riemannian one with constant sectional and scalar curvatures given by

$$
K_{i j}=z, \quad K=6 z .
$$

Through an appropriate change of coordinates [33] we find that (4.11) is transformed into the 3D Cayley-Klein metric written in terms of geodesic polar coordinates $(r, \theta, \phi)$ :

$$
\mathrm{d} s_{\mathrm{MS}}^{2}=\mathrm{d} r^{2}+\lambda_{2}^{2} \frac{\sin ^{2}\left(\lambda_{1} r\right)}{\lambda_{1}^{2}}\left(\mathrm{~d} \theta^{2}+\frac{\sin ^{2}\left(\lambda_{2} \theta\right)}{\lambda_{2}^{2}} \mathrm{~d} \phi^{2}\right) .
$$

Therefore, according to the values of $\left(\lambda_{1}, \lambda_{2}\right)$, this metric provides the $3 \mathrm{D}$ sphere $(1,1)$, Euclidean $(0,1)$, hyperbolic $(i, 1)$, anti-de Sitter $(1, i)$, Minkowskian $(0, i)$, and de Sitter $(i, i)$ spaces.

Now the MS Hamiltonian, $\tilde{H}_{z}^{\mathrm{MS}}=2 \mathcal{H}_{z}^{\mathrm{MS}}$, is written as

$$
\tilde{H}_{z}^{\mathrm{MS}}=\frac{1}{2}\left(p_{r}^{2}+\frac{\lambda_{1}^{2}}{\lambda_{2}^{2} \sin ^{2}\left(\lambda_{1} r\right)}\left(p_{\theta}^{2}+\frac{\lambda_{2}^{2}}{\sin ^{2}\left(\lambda_{2} \theta\right)} p_{\phi}^{2}\right)\right),
$$

and the four functionally independent integrals are given by (4.9) and

$$
\tilde{I}_{z}=\left(\lambda_{2} \sin \left(\lambda_{2} \theta\right) \sin \phi p_{r}+\frac{\lambda_{1} \cos \left(\lambda_{2} \theta\right) \sin \phi}{\tan \left(\lambda_{1} r\right)} p_{\theta}+\frac{\lambda_{1} \lambda_{2} \cos \phi}{\tan \left(\lambda_{1} r\right) \sin \left(\lambda_{2} \theta\right)} p_{\phi}\right)^{2},
$$

where $\tilde{I}_{z}=4 \lambda_{2}^{2} \mathcal{I}_{z}$. The two sets $\left\{\tilde{H}_{z}^{\mathrm{MS}}, \tilde{C}_{z}^{(2)}, \tilde{C}_{z}^{(3)}\right\}$ and $\left\{\tilde{H}_{z}^{\mathrm{MS}}, \tilde{C}_{z,(2)}, \tilde{I}_{z}\right\}$ consist of three functions in involution. Similarly to (4.10), this Hamiltonian is also separable and can be reduced to a 1D system:

$$
\tilde{H}_{z}^{\mathrm{MS}}\left(r, p_{r}\right)=\frac{1}{2}\left(p_{r}^{2}+\frac{\lambda_{1}^{2}}{\lambda_{2}^{2} \sin ^{2}\left(\lambda_{1} r\right)} \tilde{C}_{z}^{(3)}\right) .
$$




\section{$5 \quad$ ND spaces with variable curvature}

The generalization to arbitrary dimension is obtained through the same procedure, and the starting point is the QMS Hamiltonian for the ND geodesic motion that, in the simplest case, reads:

$$
\mathcal{H}_{z}=\frac{1}{2} J_{+}^{(N)}=\frac{1}{2} \sum_{i=1}^{N} \frac{\sinh z q_{i}^{2}}{z q_{i}^{2}} p_{i}^{2} \exp \left(-z \sum_{k=1}^{i-1} q_{k}^{2}+z \sum_{l=i+1}^{N} q_{l}^{2}\right) .
$$

The geometric characterization of the underlying ND curved spaces follows the same path as in the $2 \mathrm{D}$ and $3 \mathrm{D}$ cases described in the previous sections.

If we write the above Hamiltonian as

$$
\mathcal{H}_{z}=\frac{1}{2} \sum_{i=1}^{N} s_{z}\left(q_{i}^{2}\right) p_{i}^{2} \exp \left(z \sum_{k=1 ; k \neq i}^{N} \operatorname{sgn}(k-i) q_{k}^{2}\right),
$$

where $s_{z}\left(q_{i}^{2}\right)=\sinh z q_{i}^{2} /\left(z q_{i}^{2}\right)$ and $\operatorname{sgn}(k-i)$ is the sign of the difference $k-i$, we get again a free Lagrangian:

$$
\mathcal{T}_{z}=\frac{1}{2} \sum_{i=1}^{N} \frac{\left(\dot{q}_{i}\right)^{2} \exp \left(-z \sum_{k=1 ; k \neq i}^{N} \operatorname{sgn}(k-i) q_{k}^{2}\right)}{s_{z}\left(q_{i}^{2}\right)},
$$

with the corresponding (diagonal) metric given by

$$
\mathrm{d} s^{2}=\sum_{i=1}^{N} g_{i i}(q) \mathrm{d} q_{i}^{2}, \quad g_{i i}(q)=\frac{\exp \left(-z \sum_{k=1 ; k \neq i}^{N} \operatorname{sgn}(k-i) q_{k}^{2}\right)}{s_{z}\left(q_{i}^{2}\right)} .
$$

It turns out that the most suitable way to understand the nature of the problem as well as to enforce separability is to consider two sets of new coordinates:

- $N+1$ "collective" variables [42] $\left(\xi_{0}, \xi_{1}, \ldots, \xi_{N}\right)$. They play a similar role to the ambient coordinates arising when ND Riemannian spaces of constant curvature are embedded within $\mathbb{R}^{N+1}$.

- $N$ "intrinsic" variables $\left(\rho, \theta_{2}, \ldots, \theta_{N}\right)$ which describe the $N D$ space itself. They are the analogous to the geodesic polar coordinates on ND Riemannian spaces of constant curvature $[10,11]$.

The above coordinates are defined in terms of the initial $q_{i}$ by:

$$
\begin{aligned}
& \xi_{0}^{2}=\cosh ^{2}\left(\lambda_{1} \rho\right):=\prod_{i=1}^{N} \exp \left(2 z q_{i}^{2}\right), \\
& \xi_{k}^{2}=\sinh ^{2}\left(\lambda_{1} \rho\right) \prod_{j=2}^{k} \sin ^{2} \theta_{j} \cos ^{2} \theta_{k+1}:=\prod_{i=1}^{N-k} \exp \left(2 z q_{i}^{2}\right)\left(\exp \left(2 z q_{N-k+1}^{2}\right)-1\right), \\
& \xi_{N}^{2}=\sinh ^{2}\left(\lambda_{1} \rho\right) \prod_{j=2}^{N} \sin ^{2} \theta_{j}:=\exp \left(2 z q_{1}^{2}\right)-1,
\end{aligned}
$$


where $z=\lambda_{1}^{2}, k=1, \ldots, N-1$, and hereafter a product $\prod_{j}^{k}$ such that $j>k$ is assumed to be equal to 1 . Notice also that for the sake of simplicity we have not introduced the additional signature parameter $\lambda_{2}$ (which would have been associated with $\theta_{2}$ ). This definition is the ND generalization of the change of coordinates (4.8) given in the $3 \mathrm{D}$ case with $\theta=\theta_{2}, \phi=\theta_{3}$ and $\lambda_{2}=1$.

Clearly, the $N+1$ collective variables are not independent and they fulfil a pseudosphere relation (of hyperbolic type):

$$
\xi_{0}^{2}-\sum_{k=1}^{N} \xi_{k}^{2}=1
$$

The geodesic flow in the canonical coordinates $(\rho, \theta)$ and momenta $\left(\rho, p_{\theta}\right)$ is then given by the Hamiltonian $\tilde{H}_{z}=2 \mathcal{H}_{z}$ :

$$
\tilde{H}_{z}=\frac{1}{2} \cosh \left(\lambda_{1} \rho\right)\left(p_{\rho}^{2}+\frac{\lambda_{1}^{2}}{\sinh ^{2}\left(\lambda_{1} \rho\right)} \sum_{i=2}^{N}\left(\prod_{j=2}^{i-1} \frac{1}{\sin ^{2} \theta_{j}}\right) p_{\theta_{i}}^{2}\right),
$$

and the (left) integrals of the motion $\tilde{C}_{z}^{(m)}=4 C_{z}^{(m)}$ are found to be

$$
\tilde{C}_{z}^{(m)}=\sum_{i=N-m+2}^{N}\left(\prod_{j=N-m+2}^{i-1} \frac{1}{\sin ^{2} \theta_{j}}\right) p_{\theta_{i}}^{2}, \quad m=2, \ldots, N .
$$

By taking into account the $N$ functions $\left\{\tilde{H}_{z}, \tilde{C}_{z}^{(m)}\right\}$, we obtain the following set of $N$ equations, each of them depending on a single canonical pair, which shows the reduction of the system to a 1D problem:

$$
\begin{aligned}
& \tilde{C}_{z}^{(2)}\left(\theta_{N}, p_{\theta_{N}}\right)=p_{\theta_{N}}^{2}, \\
& \tilde{C}_{z}^{(m)}\left(\theta_{N-m+2}, p_{\theta_{N-m+2}}\right)=p_{\theta_{N-m+2}}^{2}+\frac{C_{z}^{(m-1)}}{\sin ^{2} \theta_{N-m+2}}, \quad m=3, \ldots, N, \\
& \tilde{H}_{z}\left(\rho, p_{\rho}\right)=\frac{1}{2} \cosh \left(\lambda_{1} \rho\right)\left(p_{\rho}^{2}+\frac{\lambda_{1}^{2}}{\sinh ^{2}\left(\lambda_{1} \rho\right)} \tilde{C}_{z}^{(N)}\right) .
\end{aligned}
$$

We stress that these models can be extended to incorporate appropriate interactions with an external central field, preserving superintegrability. This will be achieved by modifying the Hamiltonian by adding an arbitrary function of $J_{-}$, as we shall see in the next Section.

Finally we remark that the corresponding generalization to ND spaces with constant curvature can be obtained by considering the MS Hamiltonian

$$
\mathcal{H}_{z}^{\mathrm{MS}}=\frac{1}{2} J_{+}^{(N)} \mathrm{e}^{z J_{-}^{(N)}}=\frac{1}{2} \mathrm{e}^{z \mathbf{q}^{2}} \sum_{i=1}^{N} \frac{\sinh z q_{i}^{2}}{z q_{i}^{2}} p_{i}^{2} \exp \left(-z \sum_{k=1}^{i-1} q_{k}^{2}+z \sum_{l=i+1}^{N} q_{l}^{2}\right) .
$$

Under a suitable change of coordinates, similar to (5.2) but involving a different radial coordinate $r$ instead of $\rho$, this Hamiltonian leads to the MS geodesic motion on $\mathbf{S}^{N}, \mathbf{H}^{N}$ and $\mathbf{E}^{N}$ in the proper geodesic polar coordinates which can be found in $[10,11]$.

\section{QMS potentials}

As we have just noticed, we can also consider more general ND QMS Hamiltonians based on $s l_{z}(2, \mathbb{R})(3.7)$ by considering arbitrary $b_{i}$ 's (contained in $J_{+}$) and adding some functions depending on $J_{-}$; hereafter we drop the index " $(N)$ " in the generators. The family of Hamiltonians 
that we consider has the form (see [32] for the $2 \mathrm{D}$ construction):

$$
\mathcal{H}_{z}=\frac{1}{2} J_{+} f\left(z J_{-}\right)+\mathcal{U}\left(z J_{-}\right)
$$

where the arbitrary smooth functions $f$ and $\mathcal{U}$ are such that

$$
\lim _{z \rightarrow 0} \mathcal{U}\left(z J_{-}\right)=\mathcal{V}\left(J_{-}\right), \quad \lim _{z \rightarrow 0} f\left(z J_{-}\right)=1 .
$$

This, in turn, means that

$$
\lim _{z \rightarrow 0} \mathcal{H}_{z}=\frac{1}{2} \mathbf{p}^{2}+\mathcal{V}\left(\mathbf{q}^{2}\right)+\sum_{i=1}^{N} \frac{b_{i}}{2 q_{i}^{2}},
$$

recovering the superposition of a central potential $\mathcal{V}\left(J_{-}\right) \equiv \mathcal{V}\left(\mathbf{q}^{2}\right)$ with $N$ centrifugal terms on $\mathbf{E}^{N}$ [36]. Such a "flat" system has a (non-deformed) $s l(2, \mathbb{R})$ coalgebra symmetry as given in Theorem 1.

We recall that the function $f\left(z J_{-}\right)$gives us the type of curved background, which is characterized by the metric $\mathrm{d} s^{2} / f\left(z \mathbf{q}^{2}\right)$ where $\mathrm{d} s^{2}$ is the variable curvature metric associated to $\mathcal{H}_{z}=\frac{1}{2} J_{+}$ and given in (5.1). The two special cases with $f\left(z J_{-}\right)=\mathrm{e}^{ \pm z J_{-}}$give rise to Riemannian spaces of constant sectional curvatures, all equal to $\pm z$ (as (5.3)).

In particular, QMS deformations of the $N D \mathrm{SW}$ system would be given by any $\mathcal{U}$ such that

$$
\lim _{z \rightarrow 0} \mathcal{U}\left(z J_{-}\right)=\omega J_{-},
$$

and for the $N D$ generalized $\mathrm{KC}$ potential we can consider $\mathcal{U}$ functions such that

$$
\lim _{z \rightarrow 0} \mathcal{U}\left(z J_{-}\right)=-k / \sqrt{J_{-}} .
$$

In both cases centrifugal type potentials come from the $b_{i}$ 's terms contained in $J_{+} f\left(z J_{-}\right)$.

With account of the geometrical arguments, the following QMS SW system on spaces with non-constant curvature (5.1) has been proposed in [32]:

$$
\mathcal{H}_{z}^{\mathrm{SW}}=\frac{1}{2} J_{+}+\omega \frac{\sinh z J_{-}}{z},
$$

while a candidate for a generalized KC system on such spaces is given by the formula [32]:

$$
\mathcal{H}_{z}^{\mathrm{KC}}=\frac{1}{2} J_{+}-k \sqrt{\frac{2 z}{\mathrm{e}^{2 z J_{-}}-1}} \mathrm{e}^{2 z J_{-}} .
$$

In the constant curvature case, the approach here presented allows us to recover the known results for the SW potential on Riemannian spaces with constant curvature, as well as their generalization to relativistic spaces (whenever the signature parameter $\lambda_{2}$ is considered).

In particular, the MS SW system on ND spaces of constant curvature is given by the Hamiltonian

$$
\mathcal{H}_{z}^{\mathrm{MS}, \mathrm{SW}}=\frac{1}{2} J_{+} \mathrm{e}^{z J_{-}}+\omega \frac{\sinh z J_{-}}{z} \mathrm{e}^{z J_{-}} \equiv \mathcal{H}_{z}^{\mathrm{SW}} \mathrm{e}^{z J_{-}},
$$

and the additional constant of the motion that provides the MS property reads

$$
\mathcal{I}_{z}=\frac{\sinh z q_{1}^{2}}{2 z q_{1}^{2}} \mathrm{e}^{z q_{1}^{2}} p_{1}^{2}+\frac{z b_{1}}{2 \sinh z q_{1}^{2}} \mathrm{e}^{z q_{1}^{2}}+\frac{\omega}{2 z} \mathrm{e}^{2 z q_{1}^{2}} .
$$

The corresponding results for the generalized $\mathrm{KC}$ system are currently under investigation. 


\section{Concluding remarks}

The main message that we would like to convey to the scientific community through the present paper is that "Superintegrable Systems are not rare!". Indeed, in our approach they turn out to be a natural manifestation of coalgebra symmetry: as such, they can be equally well constructed on a flat or on a curved background, the latter being possibly equipped with a variable curvature. Moreover, and, we would say, quite remarkably the construction holds for an arbitrary number of dimensions.

In that perspective, the most interesting problems that are still open are in our opinion the following ones:

1. The explicit integration of the equations of motion for (at least some) of the prototype examples we have introduced in the previous sections;

2. The construction of the quantum-mechanical counterpart of our approach.

As for the former point, partial results have already been obtained, and a detailed description of the most relevant examples will be published soon. The latter point, in particular as far as the non-standard deformation of $\operatorname{sl}(2, \mathbb{R})$ is concerned, is however more subtle and deserves careful investigation (which is actually in progress). In fact, first of all one has to find a proper $\infty$-dimensional representation of such a non-standard deformation in terms of linear operators acting on a suitably defined Hilbert space, ensuring self-adjointness of the Hamiltonians; second, and certainly equally important, at least in some physically interesting special cases one would like to exhibit the explicit solution of the corresponding spectral problem.

\section{Acknowledgements}

This work was partially supported by the Ministerio de Educación y Ciencia (Spain, Project FIS2004-07913), by the Junta de Castilla y León (Spain, Project VA013C05), and by the INFNCICyT (Italy-Spain).

\section{References}

[1] Fris J., Mandrosov V., Smorodinsky Ya.A., Uhlir M., Winternitz P., On higher symmetries in quantum mechanics, Phys. Lett. 16 (1965), 354-356.

[2] Evans N.W., Super-integrability of the Winternitz system, Phys. Lett. A 147 (1990), 483-486.

[3] Rodriguez M.A., Winternitz P., Quantum superintegrability and exact solvability in $n$ dimensions, J. Math. Phys. 43 (2002), 1309-1322, math-ph/0110018.

[4] Kalnins E.G., Williams G.C., Miller W.Jr., Pogosyan G.S., On superintegrable symmetry-breaking potentials in $N$-dimensional Euclidean space, J. Phys. A: Math. Gen. 35 (2002), 4755-4773.

[5] Wojciechowski S., Superintegrability of the Calogero-Moser system, Phys. Lett. A 95 (1983), 279-281.

[6] Smirnov R.G., Winternitz P., A class of superintegrable potentials of Calogero type, J. Math. Phys. 47 (2006), 093505, 8 pages, math-ph/0606006.

[7] Ranada M.F., Superintegrability of the Calogero-Moser system: constants of motion, master symmetries, and time-dependent symmetries, J. Math. Phys. 40 (1999), 236-247.

[8] Gonera C., On the superintegrability of Calogero-Moser-Sutherland model, J. Phys. A: Math. Gen. 31 (1998), 4465-4472.

[9] Gonera C., Isochronic potentials and new family of superintegrable systems, J. Phys. A: Math. Gen. 37 (2004), 4085-4095.

[10] Ballesteros A., Herranz F.J., Santander M., Sanz-Gil T., Maximal superintegrability on $N$-dimensional curved spaces, J. Phys. A: Math. Gen. 36 (2003), L93-L99, math-ph/0211012. 
[11] Herranz F.J., Ballesteros A., Santander M., Sanz-Gil T., Maximally superintegrable Smorodinsky-Winternitz systems on the $N$-dimensional sphere and hyperbolic spaces, in Superintegrability in Classical and Quantum Systems, Editors P. Tempesta et al., CRM Proc. and Lecture Notes, Vol. 37, AMS, Providence, RI, 2004, 75-89, math-ph/0501035.

[12] Rañada M.F., Santander M., Superintegrable systems on the two-dimensional sphere $S^{2}$ and the hyperbolic plane $H^{2}$, J. Math. Phys. 40 (1999), 5026-5057.

[13] Kalnins E.G., Miller W.Jr., Pogosyan G.S., Completeness of multiseparable superintegrability on the complex 2-sphere, J. Phys. A: Math. Gen. 33 (2000), 6791-6806.

[14] Kalnins E.G., Kress J.M., Pogosyan G.S., Miller W.Jr., Completeness of superintegrability in twodimensional constant-curvature spaces, J. Phys. A: Math. Gen. 34 (2001), 4705-4720, math-ph/0102006.

[15] Kalnins E.G., Miller W.Jr., Pogosyan G.S., Superintegrability of the two-dimensional hyperboloid, J. Math. Phys. 38 (1997), 5416-5433.

[16] Higgs P.W., Dynamical symmetries in a spherical geometry. I, J. Phys. A: Math. Gen. 12 (1979), 309-323.

[17] Leemon I., Dynamical symmetries in a spherical geometry. II, J. Phys. A: Math. Gen. 12 (1979), $489-501$.

[18] Kalnins E.G., Miller W.Jr., Pogosyan G.S., Coulomb-oscillator duality in spaces of constant curvature, J. Math. Phys. 41 (2000), 2629-2657, quant-ph/9906055.

[19] Nersessian A., Pogosyan G., Relation of the oscillator and Coulomb systems on spheres and pseudospheres, Phys. Rev. A 63 (2001), 020103, 4 pages, quant-ph/0006118.

[20] Herranz F.J., Ballesteros A., Superintegrability on three-dimensional Riemannian and relativistic spaces of constant curvature, SIGMA 2 (2006), 010, 22 pages, math-ph/0512084.

[21] Blaszak M., Sergyeyev A., Maximal superintegrability of Benenti systems, J. Phys. A: Math. Gen. 38 (2005), L1-L5, solv-int/0312025.

[22] Ballesteros A., Herranz F.J., Integrable deformations of oscillator chains from quantum algebras, J. Phys. A: Math. Gen. 32 (1999), 8851-8862, solv-int/9911004.

[23] Kalnins E.G., Kress J.M., Miller W.Jr., Pogosyan G.S., Complete sets of invariants for dynamical systems that admit a separation of variables, J. Math. Phys. 43 (2002), 3592-3609.

[24] Kalnins E.G., Kress J.M., Miller W.Jr., Winternitz P., Superintegrable systems in Darboux spaces, J. Math. Phys. 44 (2003), 5811-5848, math-ph/0307039.

[25] Kalnins E.G., Kress J.M., Miller W.Jr., Second order superintegrable systems in conformally flat spaces. I. Two-dimensional classical structure theory, J. Math. Phys. 46 (2005), 053509, 28 pages.

[26] Kalnins E.G., Kress J.M., Miller W.Jr., Second order superintegrable systems in conformally flat spaces. II. The classical two-dimensional Stäckel transform, J. Math. Phys. 46 (2005), 053510, 15 pages.

[27] Kalnins E.G., Kress J.M., Miller W.Jr., Second order superintegrable systems in conformally flat spaces. III. 3D classical structure theory, J. Math. Phys. 46 (2005), 103507, 28 pages.

[28] Kalnins E.G., Kress J.M., Miller W.Jr., Second order superintegrable systems in conformally flat spaces. IV. The classical 3D Stackel transform and 3D classification theory, J. Math. Phys. 46 (2006), 043514, 26 pages.

[29] Kalnins E.G., Kress J.M., Miller W.Jr., Second order superintegrable systems in conformally flat spaces. V. Two- and three-dimensional quantum systems, J. Math. Phys. 46 (2006), 093501, 25 pages.

[30] Ballesteros A., Herranz F.J., Universal integrals for superintegrable systems on $N$-dimensional spaces of constant curvature, J. Phys. A: Math. Theor. 40 (2007), F51-F59, math-ph/0610040.

[31] Ballesteros A., Herranz F.J., Ragnisco O., Curvature from quantum deformations, Phys. Lett. B 610 (2005), 107-114, hep-th/0504065.

[32] Ballesteros A., Herranz F.J., Ragnisco O., Integrable potentials on spaces with curvature from quantum groups, J. Phys. A: Math. Gen. 38 (2005), 7129-7144, math-ph/0505081.

[33] Ballesteros A., Herranz F.J., Ragnisco O., Integrable geodesic motion on 3D curved spaces from non-standard quantum deformations, Czech. J. Phys. 55 (2005), 1327-1333, math-ph/0508038.

[34] Ballesteros A., Ragnisco O., A systematic construction of integrable Hamiltonians from coalgebras, J. Phys. A: Math. Gen. 31 (1998), 3791-3813, solv-int/9802008.

[35] Ballesteros A., Herranz F.J., Musso F., Ragnisco O., Superintegrable deformations of the Smorodinsky-Winternitz Hamiltonian, in Superintegrability in Classical and Quantum Systems, Editors P. Tempesta et al., CRM Proc. and Lecture Notes, Vol. 37, AMS, Providence, RI, 2004, 1-14, math-ph/0412067. 
[36] Evans N.W., Superintegrability in classical mechanics, Phys. Rev. A 41 (1990), 5666-5676.

[37] Schrödinger E., A method of determining quantum mechanical eigenvalues and eigenfunctions, Proc. R. Ir. Acad. A 46 (1940), 9-16.

[38] Ohn C., *-product on $S L(2)$ and the corresponding nonstandard quantum- $U(\mathfrak{s l}(2))$, Lett. Math. Phys. 25 (1992), 85-88.

[39] Chari V., Pressley A., A guide to quantum groups, Cambridge Univ. Press, Cambridge, 1994.

[40] Herranz F.J., Ortega R., Santander M., Trigonometry of spacetimes: a new self-dual approach to a curvature/signature (in)dependent trigonometry, J. Phys. A: Math. Gen. 33 (2000), 4525-4551, for an extended version see math-ph/9910041.

[41] Herranz F.J., Santander M., Conformal symmetries of spacetimes J. Phys. A: Math. Gen. 35 (2002), $6601-$ 6618, math-ph/0110019.

[42] Ballesteros A., Ragnisco O., Classical dynamical systems from $q$-algebras: 'cluster' variables and explicit solutions, J. Phys. A: Math. Gen. 36 (2003), 10505-10518, math-ph/0307013. 